\title{
Analysis and Examination of Heart distress from ECG signal using Artificial Intelligence.
}

\author{
Leena B. Chaudhari ${ }^{{ }^{*}}$ and Dhananjay E. Upasani ${ }^{2}$ \\ ${ }^{1}$ Bharati Vidyapeeth's College of Engineering, Lavale, Pune, India. \\ ${ }^{2}$ MIT School of Engineering, MIT ADT University, Pune, India.
}

\begin{abstract}
Health data analysis is usually based on a comparison of derived health measures to predefined thresholds. Symptoms can be observed if a value is above or below a threshold. Early detection of signs of heart failure allows the prediction of strokes of heart failure and can therefore prevent these. So identifying "accurate" criteria is the most important task. The accuracy of an experiment depends strongly on the accuracy of the criteria used. Congestive heart failure (CHF) occurs when the heart can not pump sufficient blood for a stable physiological condition. CHF usually occurs when the coronary artery blockage causes the heart tissue to become acidic. The data used to analyze data such as Linear Regression, Missing Enrollment Data, Search Signal, Clinical Data Protection Programs, and Early Adaptive Alarm. The proposed system involves models including server and data warehouse processing, pre-processing, extraction classification characterization in this paper. Classification of heart defects and prediction of heart failure by using applied classifier for hybridization, guideline for treating patients as a gym, level of stress management. In this article, the program tracks the heart disease patients, predicts atrial fibrillation and ventricular fibrillation, and alerts patients when the critical condition occurs.
\end{abstract}

\section{Introduction}

According to the World Health Organization (World Health Organization, 2016), chronic diseases such as coronary heart disease, cancer, chronic obstructive pulmonary disease and type 2 diabetes mellitus are the world's leading cause of death, accounting for around $60 \%$ of all deaths. Chronic diseases are due mainly to heart failure, currently the leading cause of death in most western countries. The American Heart Heart Association (AHA) 2016 Heart Disease and Stroke Statistics update estimated that 15.5 million people in the United States suffer from cardiovascular disease, a prevalence that is growing with age for both men and women [1]. Throughout Europe, the predominance of individuals describing heart problems for both sexes was 9.2 per cent over the last year [2]. Cardiovascular diseases are the main cause of death in Italy in particular, accounting for 44 per cent of all deaths. Cardiovascular disease passages in India grew from 1.3 million in each of 1990 to 2.8 million in 2016, and most of the passages were caused by heart disease.

Social insurance is a pending problem with worldwide difficulties[3 ]. The acute socio-economic situation confronting a significant portion of developed countries is generating greater pressure to find more costeffective solutions to health and social care delivery. In addition, healthcare systems in developing countries face serious difficulties in providing care and assistance, primarily due to a lack of staff and resources. Over the last few years a few proposals have emerged as a part of the new period of human services. Drug 2.0, Wellness 2.0/3.0, ePatient, and eDoctor are usually dispersed examples of such terms, among other commonly established words such as eHealth, telehealth, or telemedicine. Likewise, frameworks might be utilized in a pervasive way and give consistent observing abilities.One of the major disadvantages of the up-to-date portable health monitoring tools from a technical perspective is the processing and storage capacities. Checking gadgets consist regularly of many sensors which measure physiological sizes and convert them into discernible machine data [4]. While some of these devices contain dedicated resources for processing the information, they are very sparse and limited. Although some of these devices contain dedicated information processing tools, they are very sparse and minimal. On the other hand, mobile devices are the perfect way to collect data from health monitoring systems, provide local processing capabilities or even provide access to cloud services (high-performance computing, large capacity storage, and data analytics) [5] . Through this, not only the data collected from the patient could be taken into account for their health assessment and treatment, but also the records of hundreds, thousands or even millions of patients, which might share similar needs and conditions [6].

Atrial fibrillation (AF) has been depicted by doctors as the commonest heart arrhythmia in clinical everyday

*Corresponding author: leenapc23@gmail.com 
practice, with an expected predominance of $1.5 \%-2 \%$ of the overall public in the created world [7]. In excess of $6,000,000$ individuals in Europe and 3,000,000 individuals in the USA experience the ill effects of this arrhythmia [7]. It is additionally expected that its predominance will twofold in the following 50 years [8]. Today, three distinct sorts of AF are clinically stratified relying upon the scene length. The arrhythmia would then be able to be grouped into paroxysmal $\mathrm{AF}$ (PAF), persevering or long-standing tenacious AF and, at long last, perpetual AF . Indeed, the nearness of AF is related with a five-crease danger of stroke and a three-overlay frequency of congestive cardiovascular breakdown, accordingly inciting that AF patients have double the danger of death than sound individuals of a similar age [7]. Inside this specific situation, an early discovery of AF may help with decreasing that hazard by reestablishing typical heart mood or by improving the blood stream with antithrombotic treatment. This early finding may likewise include prominent advantages for social insurance benefits the world over, in light of the fact that the high hospitalization paces of AF, just as its extensive weight on wellbeing assets could be fundamentally restricted - Cardiovascular sign from both surface and intracardiac accounts have been generally utilized in AF thinks about planning to comprehend the atrial electrical conduct to improve adequacy of interventional removal treatment, however the nearness of ventricular movement (VA) on these sign has hampered the investigation with probability of mutilated outcomes. It is imperative to think about atrial action (AA) without the impact of the VA to improve comprehension of AF beginning and support [9].

Ventricular fibrillation is the quick, disarranged, and offbeat withdrawal of ventricular muscle. Hoffa and Ludwig recommended in 1850 that the inconsistency of these developments is on the grounds that the individual anatomical components lose their interrelationship and quit contracting as one. In 1887, MacWilliam depicted "a condition of fibrillar compression comprised by a quick progression of in facilitated peristaltic constrictions, and in 1914, utilized the term ventricular fibrillation to portray what he thought about the reason for unexpected passing. Superficially electrocardiogram, ventricular fibrillation is portrayed by the nonappearance of unmistakably characterized QRS edifices. It frequently happens in patients with auxiliary heart variations from the norm. Be that as it may, in certain patients, show coronary illness is missing, and the reason for the arrhythmia can't be distinguished or must be learned by broad assessment (e.g., hereditary investigation). Incredible consideration has been given to ventricular fibrillation since it speaks to the last basic pathway for death in many patients who experience out-of-clinic heart failure, and its pace of repeat is about $30 \%$ in the primary year in effectively revived patients. Since the particular reasons for ventricular fibrillation are examined in different sections, this section gives a progressively broad outline of the clinical issue and its administration. Ventricular fibrillation (VF) is a significant reason for dismalness and abrupt passing (SD), yet it is one of the most troublesome arrhythmias to viably treat. The implantable cardioverter defibrillator (ICD) offers delayed endurance by ensuring against death because of arrhythmia and is the best quality level treatment for essential and auxiliary anticipation for in danger patients. Be that as it may, gadget implantation doesn't influence the fundamental substrate. Moreover, up to $20 \%$ of patients with an ICD experience repetitive scenes of VF or electrical tempests, bringing about numerous gadget treatments, which fall apart the nature of lifel as well as increment related mortality. Antiarrhythmic medications, for example, amiodarone, $\beta$ blockers, and lidocaine have been utilized forever undermining arrhythmias, including VF, yet their adequacy has not yet been exhibited on an enormous scale. In addition, they are related with different symptoms, which frequently exceed the questionable prognostic advantage.

\section{Objectives of the Study}

1. To develop system for individuals tormented by chronic diseases that modify the gathering, sharing and exchange of physiological knowledge, like pressure level, heart and respiration rate and cardiogram signals.

2. To predict heart disease strokes by police investigation connected symptoms.

3. To give notice the specialist and therefore the patient once a heart disease stroke has been foreseen.

4. To work out mechanically and update thresholds in keeping with recorded historical knowledge.

5. To test rules and compare the value to predefined threshold of each derived health indicator.

6. Updating threshold values according to requirement.

7. To do the review and save the original files, which can be accessed and sent to the medical specialist whenever the user wants.

\section{Approach of the study}

\subsection{Quantitative approach}

- Constant Mathematical Model: The consistent work could be a work whose esteem is the same for each input esteem.

- Step Function Mathematical Model: The constant perform may be a perform whose price is that the same for each input price.

- Average and Confidence Level Mathematical Model: A simple method to update thresholds that comes back to adding the average of $\mathrm{p}$ past data values to the confidence level of this average.

- Moving Average (MA): MA is one of the widely known technical indicators used for time series analysis. During its development, many variations and implementations 
have been proposed by researchers. Basic type of moving average is the Simple Moving Average (SMA).

\subsection{Qualitative approach}

- Allow physiological data, such as blood pressure, heart and respiration rate and ECG signals, to be recorded, transmitted, and exchanged.

- The audit report and the original files are saved, can be accessed and sent to the medical specialist whenever the user wants them to.

\section{Architecture}

In this design, the limit esteems are consequently processed by utilizing factual methodologies. The produced edges rely upon the patient state and his/her authentic estimations. The thought comprises of recognizing cardiovascular breakdown manifestations and thusly foreseeing basic circumstances (i.e.,strokes).

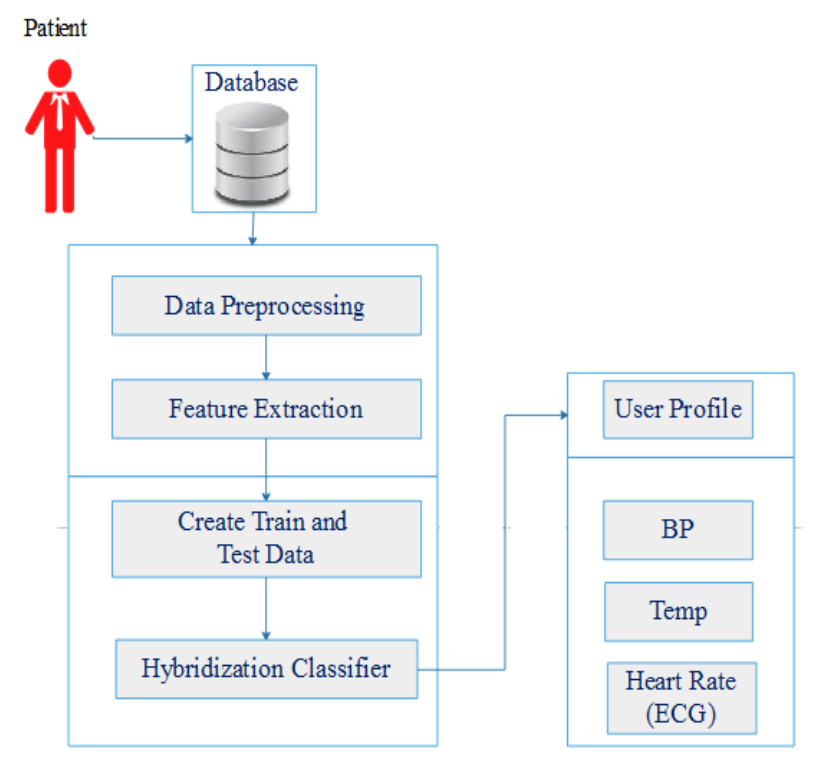

Fig. 1. Proposed System Architecture

1) Dataset: In this architectural study, the MIMIC II waveform database can be used. This is in line with the subgroup from the Psionet Benchmark, which is available online, and is collected from bed patient monitors in the adult and neonatal ICU (intensive care units).

2) First, the device stores data, such as CSV files, in a database. So, patient history is established.

3) Data is preprocessed in the preprocessing stage and the replacement values with threshold values or None are lackinga.

4) The machine performs the extraction process of the element once the preprocessing is completed. The values of the data function are determined using methods of main component analysis. The PCA's goal is to cut down on data and rate columns with a high impact point. This eliminated characteristics from this column after choosing heavily impacted columns such as weight, blood pressure, body temperature.

5) Then build the training and test files using extracted features and use implemented hybridization classifiers to perform classification. The system detects Ventricular Fibrillation and Atrial Fibrillation.

6) Finally, this program suggests care for patients such as Gym, the control of stress levels.

\section{Scope}

The program can be used to predict strokes that cause heart failure. By frequent monitoring along with support and suggestions it can be helpful for CHF patients. Patients with cardiovascular system disorders may measure their weight, blood pressure, activity, and other factors related to the health.

\section{Advantages}

1) Patients with heart disease are tracked by the machine.

2) Predicts ventricular and atrial fibrillation.

3) Warns patients when the situation is serious.

4) Automatically calculates and updates thresholds based on historical data collected.

\section{Conclusion}

Develop system for people suffering from chronic diseases. Rules are based on discussion with cardiologist and comparing extracted parameters with threshold. Feature values of data are calculated using the principal component analysis (PCA) techniques. Rules are based on discussion with cardiologist and comparing extracted parameters with threshold. This technique can be used to the prediction of Atrial Fibrillation and Ventricular Fibrillation.

\section{References}

1. Md Saiful Islam, Md Mahmudul Hasan, Xiaoyi Wang , Hayley D. Germack, and Md Noor-E-Alam, Healthcare 2018, 6, 54, (2018).

2. Afef Mdhaffar, Ismael Bouassida Rodriguez, Khalil Charfi, Leila Abid, and Bernd Freisleben, IEEE TRANSACTIONS ON NANOBIOSCIENCE, VOL. 16, NO. 8, (2017)

3. Salvatore Naddeo, Laura Verde, Manolo Forastiere, Giuseppe De Pietro and Giovanna Sannino, SCITEPRESS Science and Technology Publications, (2017).

4. A. Dhillon, S. Majumdar, M. St Hilaire and A. El. Haraki, Conference: IEEE International Conference on Internet of Things, (2017). 
5. Sebastian D. Goodfellow, Andrew Goodwin, Robert Greer, Peter C. Laussen, Mjaye Mazwi, Danny Eytan, Computing in Cardiology 2017; VOL 44

6. Francesco Nocera, Tommaso Di Noia, Marina Mongiello, Eugenio Di Sciascio, SEMAPRO 2016 : The Tenth International Conference on Advances in Semantic Processing, (2016).

7. A. Javari and M. Jalili, Knowledge Information System, volume-44, no.3, (2015).

8. Y. Rao, N. Zhang, and H. Zou,Knowledge Information System, volume. 44, No. 3, PP. 663688, (2015).

9. R. Pathak and V. Vaidehi, in Proc. 3rd Int. Conf. Eco-Friendly Comput. Commun. Syst. (ICECCS), pp. 61-66,( Dec. 2014). 